\title{
STEPHEN HAWKING: SPACE, TIME AND QUANTA
}

\author{
MAURO CARFORA $(*)$ \\ Nota presentata dal m.e. Giacomo Mauro D’Ariano \\ (Adunanza del 12 aprile 2018)
}

Sunto. - Una breve introduzione alla figura scientifica di Stephen Hawking e ai suoi contributi alla comprensione dell'interazione fra relatività generale e teoria quantistica.

$$
* * *
$$

Abstract. - A brief introduction to the scientific work of Stephen Hawking and to his contributions to our understanding of the interplay between general relativity and quantum theory.

\section{INTRODUCTION}

The scientific life of Stephen Hawking follows a path that eventually led him to establish some of the most relevant theoretical results in general relativity, from the properties of black holes and spacetime singularities, to the introduction of the revolutionary circle of ideas and techniques that go under the name of Hawking (Black Hole) Radiation. This is arguably the most significant result in the still ongoing attempt to reconcile general relativity with quantum theory, an attempt which, in the words of Roger Penrose, represents the most fundamental unsolved foundational issue in Physics.

(*) Department of Physics, University of Pavia. Italian National Group of Mathematical Physics, and INFN Pavia Section, Italy. E-mail: mauro.carfora@unipv.it 


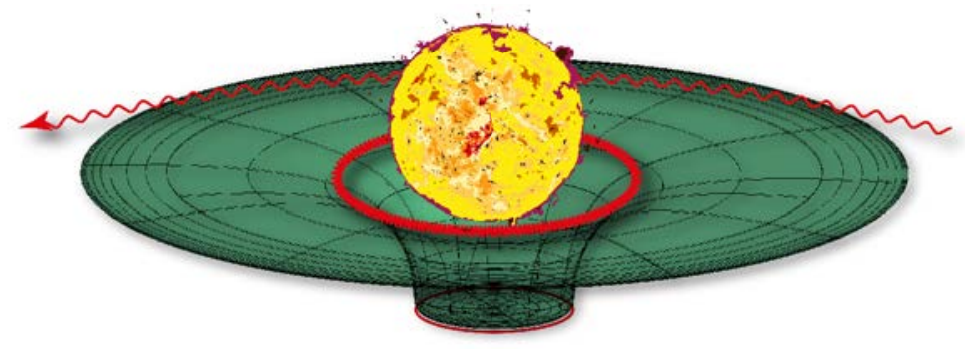

Fig. 1 - The bending of light caused by the curved geometry of space.

\section{Gravity}

Our story begins with gravity, the weakest among the known interactions, but the most evident because it is universal: gravity acts in the same manner on every form of mass-energy, hence its effects are cumulative. Moreover, gravity is a force which affects every particle in the same way, as first recognized by Galileo, a basic observation which expresses the universality of free fall: All free-falling bodies with negligible gravitational self-interactions follow the same path if they have the same initial velocities. This is the weak Principle of Equivalence which allows us to eliminate the local gravitational field. Stated more precisely, in Newtonian mechanics we have the freedom of choosing at any point (and at a given instant of time) a (non-rotating) local frame of reference in which the gravitational field, at the given point and at the given instant, can be eliminated and in which Newton equations of motion take the form they have in an inertial frame. This cannot be a peculiarity of the laws of Newtonian mechanics and with a bold step Einstein extended this property, with his Equivalence Principle, to all physical laws: In all non-rotating free-falling local frames of reference: the so-called Local Inertial Frames $(L I F))$, the non-gravitational laws of physics take the same form they have, in absence of gravity, in the inertial frames of Minkowski spacetime. In other words, non-gravitational physics in each local inertial frame is the physics of special relativity in absence of gravity, in particular in a LIF light propagates in vacuum as in special relativity. It follows that around a gravitating source light propagation is described by the collection of $l o-$ cal light cones associated with the local inertial frames surrounding the 


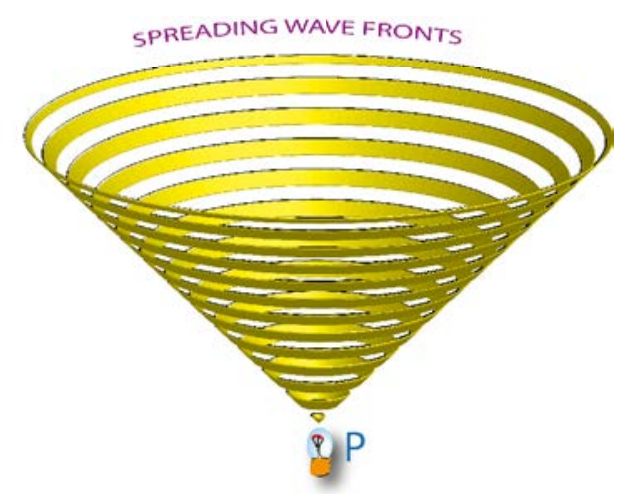

Fig. 2 - The future light cone as a collection of spreading wave fronts issuing from an event.

gravitational source: Gravity bends light (both via the geometry of time and, in a more significant way, via the geometry of space) (Fig. 1).

In a definite sense, the basic role of the light cone and causality in general relativity is the key for understanding Hawking's results. Let us recall that the future light cone at an event $P$ may be thought of as the world-history of the propagation of a light-front emitted (in vacuum) by a light-flash at P. Each light-front is a 2-sphere expanding from P. Similarly, the past light cone at $\mathrm{P}$ is the world history of a light front converging into $\mathrm{P}$ (Fig. 2).

It is worthwhile to stress that the light cone is not a peculiarity of electromagnetism. The propagation of every massless field is characterized by this propagative cone, and its structure is a property of Minkowski spacetime. The causality principle together with special relativity implies that no physical signal can travel faster than light. It follows that the light cone determines the causal structure: how spacetime events are causally related to each other (Fig. 3).

Hence, regardless of electromagnetism, the light cone can be identified as the characterization of the causal structure of Minkowski spacetime: given an event $\mathrm{P}$, it tells us which event is in the past, in the future, or in the present of P. In special relativity the light cones are all equivalent: we can map one into any other via (active) Poincaré transformations. Hence, the causal structure of Minkowski spacetime is rigid. However, if we switch on gravity this is no longer true: the local inertial frames associ- 


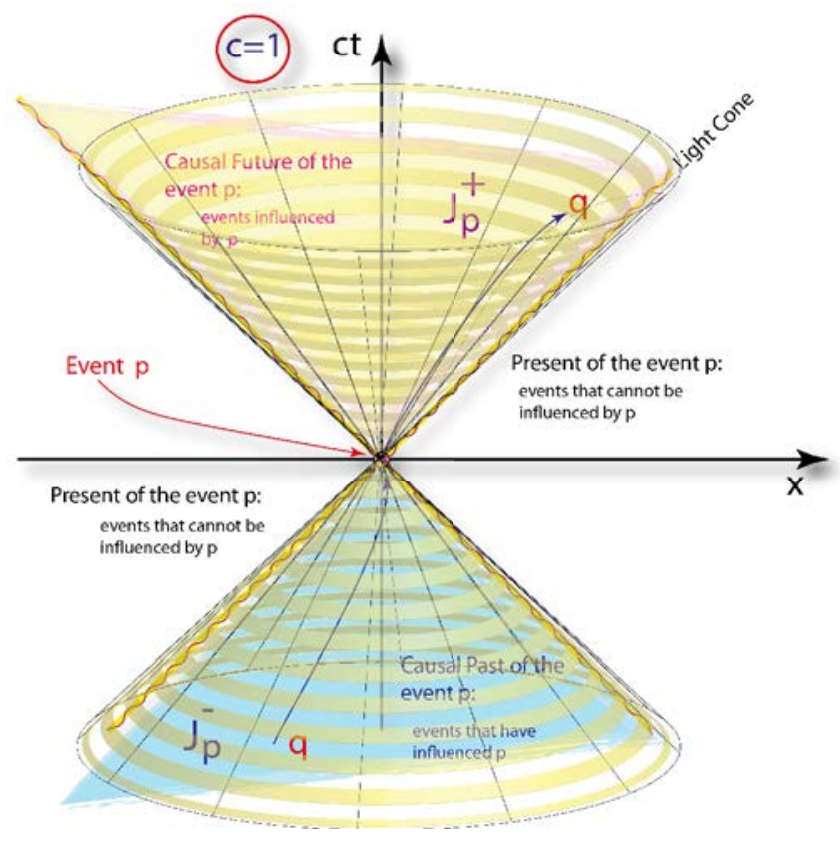

Fig. 3 - The light cone at the event $p$ and the associated causal structure.

ated with two distinct events $p$ and $q$ nearby a gravitating mass accelerate (Fig. 4) one with respect to the other as a consequence of the local gradient of the gravitational field (tidal gravitational forces). The corresponding local light cones $\mathcal{C}_{p}$ and $\mathcal{C}_{q}$ are no longer congruent one to the other. It follows that gravity deforms (and determines) the causal structure of spacetime! The gravitational interaction forces us to a shift from the flat Minkowski spacetime, as the arena for (nongravitational) physics, to a curved Lorentzian manifold $(M, g)$, where gravity is no longer a force but simply the manifestation of the dynamics of a curved spacetime (Fig. 5). This is General Relativity, the modern theory of gravitation governed by Einstein's equations,

$$
\operatorname{Ric}(g)-\frac{1}{2} g R(g)=\frac{8 \pi G}{c^{4}} T
$$

where $\operatorname{Ric}(g)$ and $R(g)$ respectively are the Ricci and the scalar curvature associated with the spacetime metric $g, T$ is the energy-momentum 


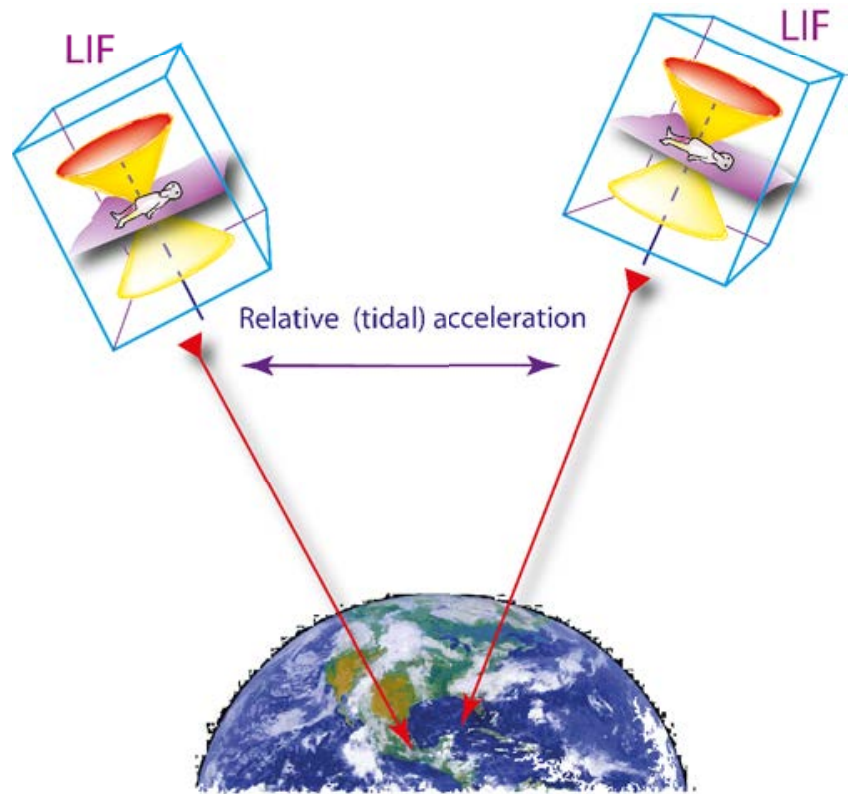

Fig. 4 - The relative acceleration between two local inertial frames generated by the gradient of the gravitational field (Tidal forces).

tensor describing the mass-energy sources present, $G$ denotes the gravitational constant and $c$ is the speed of light in vacuum. Notice the intrinsic non-linearity of the theory. Via the coupling $\frac{8 \pi G}{c^{4}}$, the distribution of mass-energy $T$ generates spacetime curvature. Conversely, spacetime curvature determines the evolution of the mass-energy sources via the freely-falling condition $\operatorname{div} T=0$, consequence of the contracted Bianchi identity div $\left(\operatorname{Ric}(g)-\frac{1}{2} g R(g)\right) \equiv 0$, (where div is the tensorial divergence $\nabla_{a} T^{a b}$ associated with the Levi-Civita connection $\nabla$ on the spacetime $(M, g))$. This non-linearity is the basic reason why Einstein theory is so complex and rich.

\section{BLACK HOLES}

The connection between spacetime geometry, causality and gravity is powerful but also leads to a severe problem: Gravitational Collapse and Singularities. In this connection, it is worthwhile to go through a back-of- 

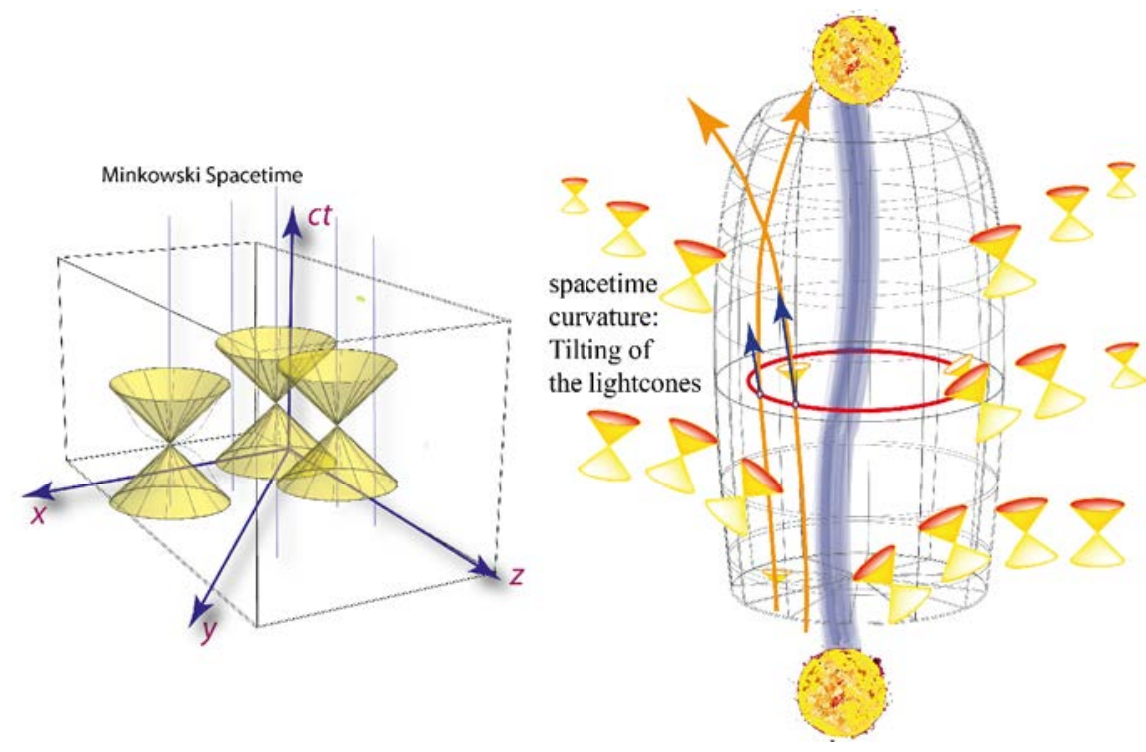

Fig. 5 - The causal structure in a curved spacetime is no longer rigid as in Minkowski spacetime: the lightcones react to the distribution and the evolution of the mass-energy present.

the-envelope calculation providing us a rough indication of the physical regime in which general relativity is relevant. From Newton theory, we know that gravity plays a significant role in the analysis of a physical phenomenon if the typical velocities $v$ induced on a test particle by the gravitational attraction exerted by a mass $M$, concentrated in a region of radius $r$, are such that

$$
\frac{1}{2} v^{2} \simeq \frac{G M}{r} .
$$

On the other hand, special relativity plays a significant role if the velocities involved in discussing a physical configuration are such that they approach, in modulus, the speed of light in vacuum, $|v| \simeq c$. It follows that general relativity is expected to play a significant role if $\frac{1}{2} c^{2} \simeq \frac{G M}{r}$, i.e., if the region where $M$ is concentrated has a radius

$$
r \simeq \frac{2 G M}{c^{2}}:=r_{S c h w}
$$




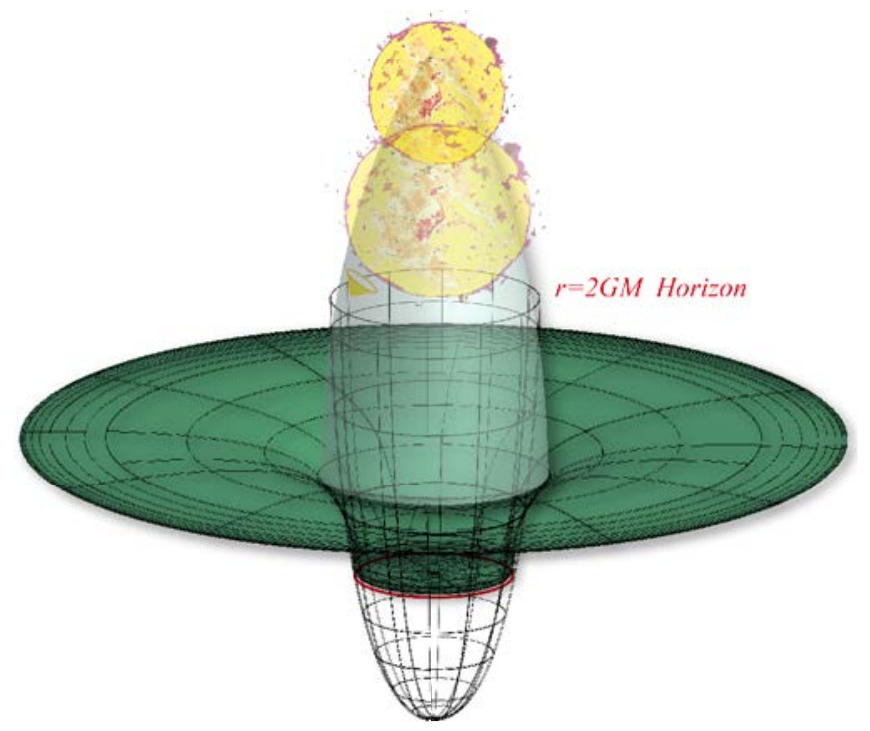

Fig. 6 - As the star evolves it may collapse under its own gravity. The more it gets concentrated in small regions the stronger are general relativistic effects. The Schwarzschild radius associated with the given mass of the star is the typical length scale signalling the relevance of general relativity in the modeling the gravitational dynamics of the star.

a quantity known as the Schwarzschild radius (Fig. G) associated to the mass $M$. This general relativistic regime is put to the fore when a star collapses under its own gravity and evolves into a Black Hole: here the connection between causal structure and gravity features at its peak. It is well-known that the possibility of the existence of Dark Stars (black holes) had been already explored in Newtonian theory by John Michell (1783) [9], and Pierre-Simon de Laplace (1798) [8]. In the very words of J. Michell (paragraph 29 of his paper [9])

If there should really exist in nature any bodies, whose density is not less than that of the sun, and whose diameters are more than 500 times the diameter of the sun, since their light could not arrive at us; of if there should exists any other bodies of a somewhat smaller size, which are not naturally luminous; of the the existence of bodies under either of these circumstances, we could have no information from light; yet, if any other luminous bodies should happen to revolve about them we might still perbaps from the mo- 


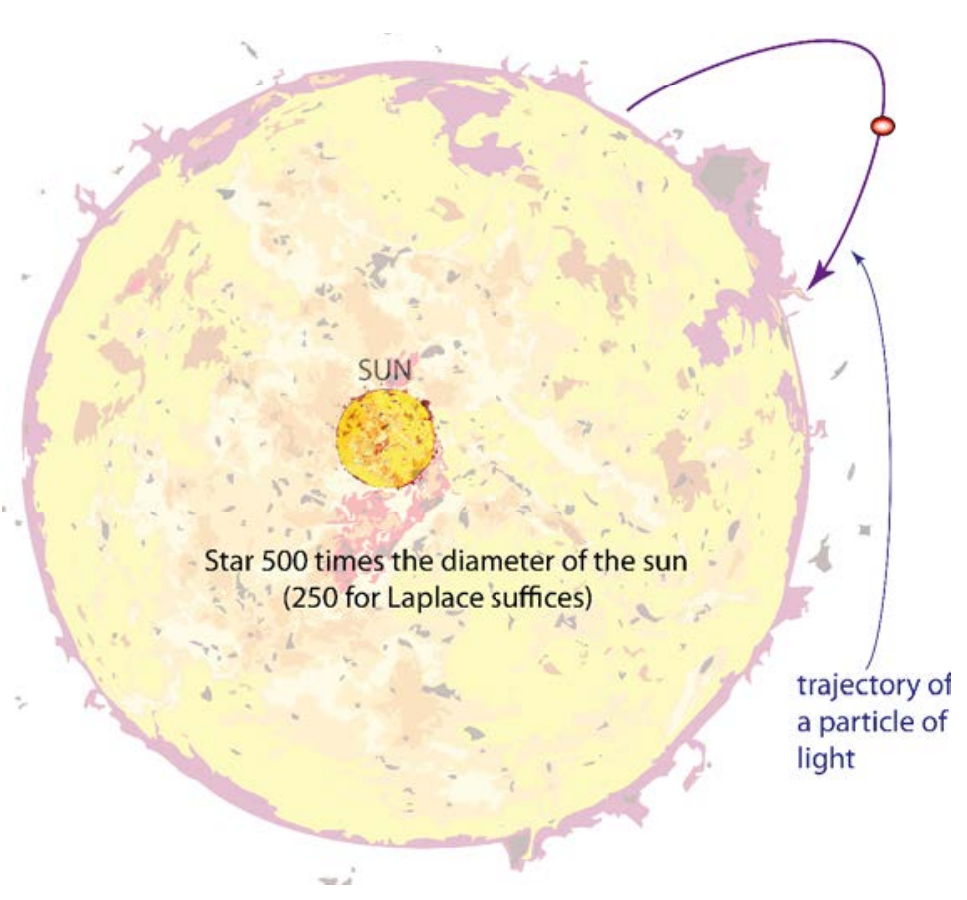

Fig. 7 - The relevant parameter for the existence of black stars in Newtonian theory is the total mass of the star rather than the density.

tions of these revolving bodies, which would not be easily explicable on any other hypothesis ... (Fig. 7)

This Newtonian result, which seems to imply that "the largest bodies in the Universe could remain invisible to us", is not dynamically very relevant, since it depends only on the mass of the star. However, according to general relativity, what matters is the dynamical concentration of a sufficiently large amount of matter in a sufficiently small region as to deflect light going out from the region so much that it can be dragged back inwards. This situation implies that matter, which cannot travel faster than light, is trapped in a region whose boundary decreases to zero within a finite proper time. A Black Hole is born, a spacetime singularity must occur (Fig. 8). The prescient work (1939) by Robert Oppenheimer and Hartland Snyder [10], describing the gravitational collapse of a ball of dust, was conducive to such a conclusion, but it was (erroneously) be- 


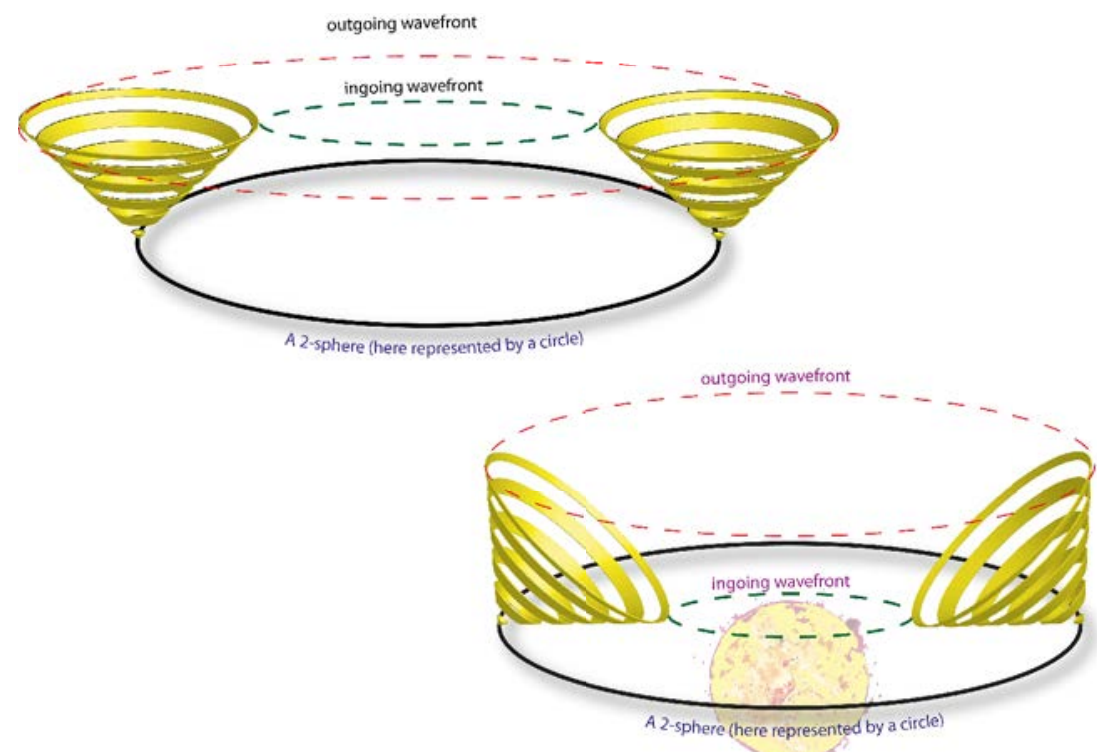

Fig. 8 - The presence of mass-energy concentrated in a given region deforms the distribution of spherical wavelets the envelope of which generates the ingoing and the outgoing wavefronts associated with the boundary of the region considered. When there is enough matter concentrated in the given region, the two families of future-pointing light rays emerging orthogonally from the boundary of the region considered are both (initially) convergent: a trapped surface has been generated.

lieved that the development of a singularity was an artifact of the spherical symmetry. That the onset of a singularity is a general consequence of gravitational collapse was proven in 1965 by R. Penrose in a remarkable paper [11] which laid the foundation of all subsequent work (and in particular of Hawking's work) on singularity theorems in general relativity. Penrose made use of modern techniques of differential geometry characterizing the spacetime singularities in terms of geodesic incompleteness, stressed the role of Cauchy hypersurfaces and introduced the notion of closed trapped surface, a two-dimensional closed surface which happens to be embedded in the given spacetime in such a way that the two families of future-pointing light rays emerging orthogonally from it are (initially) convergent (Fig. 8).

A fundamental example of the complex causal structure associated 


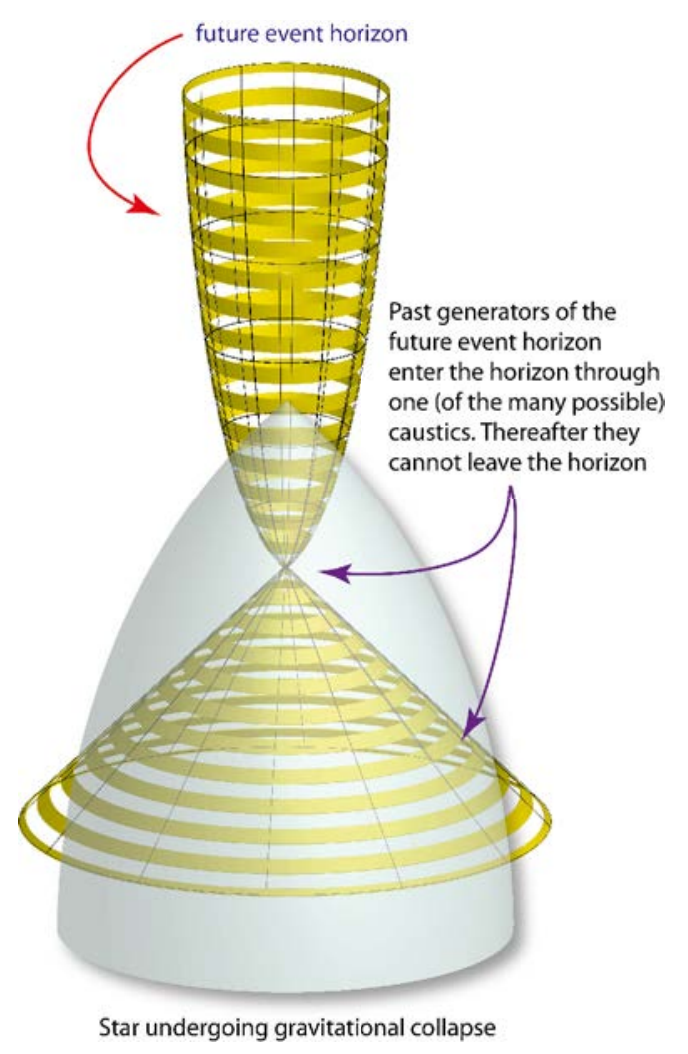

Fig. 9- The generation of the future event horizon in a collapsing star.

with a radially collapsing star eventually evolving into a non-rotating black hole (Fig. 9 and Fig. 10) is provided by Schwarzschild spacetime $(M, g)$,

$$
\begin{aligned}
g= & -\left(1-\frac{2 G M}{c^{2} r}\right) d t^{2}+\left(1-\frac{2 G M}{c^{2} r}\right)^{-1} d r^{2} \\
& +r^{2}\left(d \theta^{2}+\sin ^{2} \theta d \phi^{2}\right)
\end{aligned}
$$

This is the solution of Einstein equations in vacuum $(\operatorname{Ric}(g)=0)$ obtained, as early as 1916, by Karl Schwarzschild [13], and which geometrically describes an asymptotically Minkowskian spacetime endowed with spherical symmetry. A full understanding of the causal structure of this spacetime had to wait the 60 s (after a long chain of contributions marked 


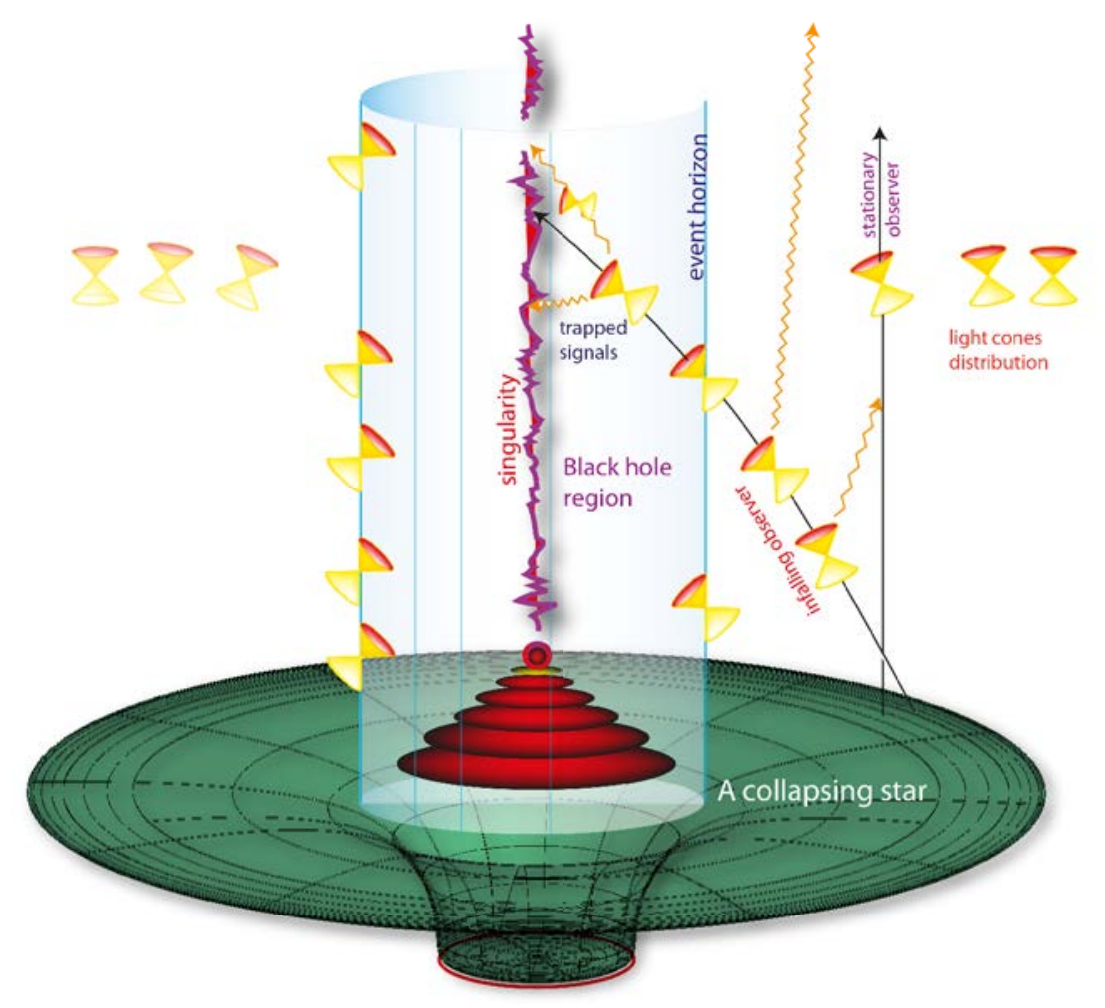

Fig. 10 - Schwarzschild spacetime describing a non-rotating black bole of mass $M$.

by G. Lemaitre, A. Eddington, J. L. Synge, M. Kruskal and G. Szekeres) when the nature of the formal singularity at the Schwarzschild radius $r=r_{S c h w}=2 G M / c^{2}$ was eventually understood. The Schwarschild metric exhibits the relevant and typical structure that are believed to characterize the spacetime region exterior to a (non-rotating) gravitationally collapsing star, namely: (i) The onset of an event horizon, precluding to the external observer access to the information on what happens to the star once its surface crosses the horizon; and (ii) the development of a singularity (which remains invisible to the external observer) into which the star inevitably falls according to general relativity. The causal structure of a Schwarzschild black hole can be clearly seen in the diagram described in Fig. 11, where the nature of the event horizon associated with the black hole region is detailed. A basic role in this discussion about black holes is 

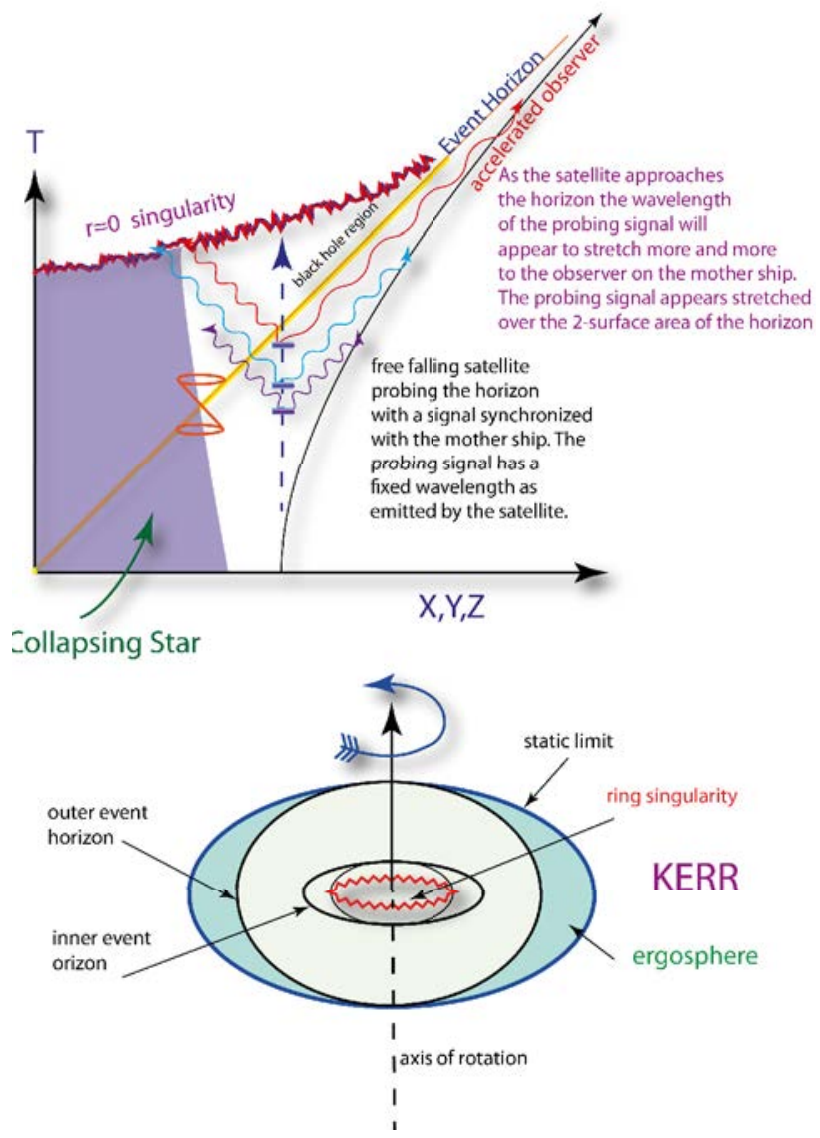

Fig. 11 - A description of the causal structure near the horizon of a Schwarzschild black bole (top) and a rotating Kerr black bole(bottom).

also played by the Kerr metric [7] (here in Boyer-Lindquist coordinates and $c=1$ )

$$
\begin{aligned}
& g=-d t^{2}+\frac{r^{2}+a^{2} \cos ^{2} \theta}{r^{2}-2 G M r+a^{2}} d r^{2}+\left(r^{2}+a^{2} \cos ^{2} \theta\right) d \theta^{2} \\
& +\left(r^{2}+a^{2}\right) \sin ^{2} \theta d \phi^{2}+\frac{2 G M r}{r^{2}+a^{2} \cos ^{2} \theta}\left(a \sin ^{2} \theta d \phi-d t^{2}\right),
\end{aligned}
$$


which describes the vacuum spacetime associated to a rotating axiallysymmetric black hole of mass $M$ and angular momentum $J=M a$. Its causal structure is described, in a sketchy form, in Fig. 11. Besides the presence of inner singularities (with a structure different from the Schwarzschild singularity) and event horizons it is characterized by the presence of a spacetime region, the ergosphere, external to the black hole's (outer) event horizon, where no time-like observer could remain stationary. Roger Penrose [12] showed that particles within this ergosphere region could possess negative energy as measured by an observer at infinity. When these particles are captured by the horizon, we can extract energy and angular momentum from the rotating black hole, a possibility that played a basic role in the development of the circle of ideas leading to the Hawking radiation.

\section{Singularities}

The basic question around the nature of Schwarzschild and Kerr black holes concerned the possibility that the development of an event horizon and of the ensuing singularities was not a generic fact, to be expected in the physical evolution of a collapsing star, but rather the consequence of the particular properties of these very symmetrical solutions of the Einstein equations. This was not an academic question, since the development of singularities in spacetime implies a corresponding break down of the laws of physics, a basic issue in modern general relativity. The two physical situations where singularity of this type occur are the gravitational collapse of sufficiently massive stars and the big bang singularity in cosmology. The problem of the cosmological singularity marked Hawking's interest in the matter. Together with G. F. R. Ellis he discussed the development of singularities in homogeneous cosmological models [1], and together (1973) they wrote the wonderful The large scale structure of Space-Time [5], where they provide a detailed and still very actual description of the sophisticated global geometrical methods pioneered by them and Roger Penrose. This classic book marked also the birth of modern mathematical general relativity. A series of events, fostered by Dennis Sciama, led to a collaboration with R. Penrose [2]. Together, they were able to prove that a big bang singularity was also a feature not just of the standard highly symmetrical Friedmann-Lemaitre-Robertson-Walker (FLRW) cosmological models, but also of more generic (not symmetric) model, a theorem generalizing Penrose's original results by showing that 


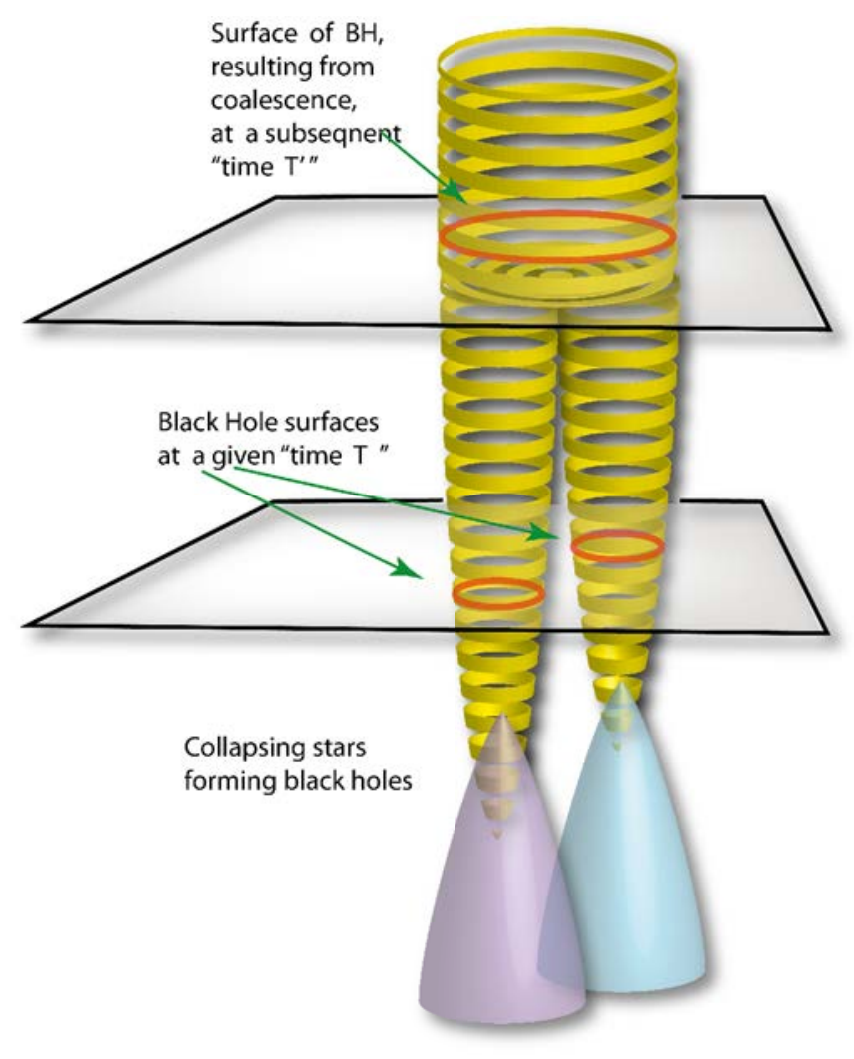

Fig. 12 - The dynamics of the event borizon resulting from the coalescence of two black boles.

a big-bang-type singularity was a necessary implication of general relativity. These years mark also Hawking's fundamental contribution [3] to a basic result of black hole theory according to which (i) The event horizon of a black hole has the topology of a sphere, (ii) The surface area of the future event horizon never decreases with time (Fig. 12). These properties suggested him a deep analogy between the behavior of black holes and the laws of thermodynamics: The surface area of the black hole horizon and its surface gravity were shown (in a foundational work [4] written in collaboration with B. Carter and J. Bardeen) to play a role analogous, respectively, to the role that entropy and temperature play in thermodynamics. 
By deriving an expression for the mass of a stationary axisymmetric solution of the Einstein equations containing a black hole surrounded by matter and discussing aslo a formula for the difference in mass between two neighboring such solutions, they noticed that two black hole parameters, the area $\mathrm{A}$ of the event horizon and the surface gravity $k$ of the black hole, have in their words a close analogy with entropy and temperature respectively. This analogy suggests the formulation of four laws of black bole mechanics which correspond to and in some ways transcend the four laws of thermodynamics (Fig. 13). In the notation associated with the Kerr metric (recall that the surface gravity of a black hole is, roughly speaking, the acceleration required (as measured from an asymptotically Minkowskian observer), for a test particle to stay still on the event horizon), we have

- Oth Law: the surface gravity of the (future) event horizon $\kappa$ is constant

$$
\kappa:=\frac{\sqrt{G^{2} M^{2}-a^{2}}}{2 G M\left(G M+\sqrt{G^{2} M^{2}-a^{2}}\right)}
$$

- 1st Law: if a stationary black hole of mass $M$ and angular momentum $J=M a$ is perturbed to another stationary $B H(M+$ $\delta M, J+\delta J)$ then

$$
d M=\frac{\kappa}{8 \pi} d A+\Omega_{H} d J
$$

where $\Omega_{H}=\frac{J}{2 G M\left[G M^{2}+\sqrt{G^{2} M^{4}-J^{2}}\right]}$ is the black hole horizon angular velocity;

- 2nd Law: Hawking's area theorem holds: The surface area of the future event horizon never decreases with time.

This thermodynamic behavior of black holes seemed, at the time, just a curiosity and we let $\mathrm{S}$. Hawking comment on this:

..But it was Jacob Bekenstein, who took the bold step, of suggesting the area actually was the physical entropy, and that it counted the internal states of the black hole. I was very much against this idea at first, because I felt it was a misuse of my horizon area result. If a black bole bad a physical entropy, it would also have a physical temperature. If a black bole was in contact with thermal radiation, it would absorb some of the radiation, but it would not give off any radiation, since by definition, a black bole was a region from 


\begin{tabular}{|c|c|c|}
\hline Law & Thermodynamics & Black Holes \\
\hline Oth & $\mathrm{T} \begin{array}{l}\text { constant for } \\
\text { system in thermodynamical } \\
\text { equilibrium }\end{array}$ & $K \begin{array}{l}\text { Constant on stationary } \\
\mathrm{BH} \text { horizon }\end{array}$ \\
\hline $1 \mathrm{st}$ & $d E=T d S+p d V$ & $d M=\frac{\kappa}{8 \pi} d A+\Omega_{H} d J$ \\
\hline 2nd & $\delta S \geq 0 \begin{array}{l}\text { in every } \\
\text { process }\end{array}$ & $\delta A \geq 0 \begin{array}{l}\text { in every } \\
\text { process }\end{array}$ \\
\hline $3 r d$ & $\begin{array}{l}\text { Impossible to get } T=0 \\
\text { in a physical process }\end{array}$ & $\begin{array}{l}\text { Impossible to get } \\
\text { in a physical process }\end{array} \quad K=0$ \\
\hline
\end{tabular}

Fig. 13 - The correspondence between the laws of thermodynamics and the laws of black bole mechanics.

which nothing would escape. If thermal radiation was at a lower temperature than the black bole, the loss of entropy down the black hole, would be greater than the increase of horizon area. ... No one, including Bekenstein and myself, thought anything could get out of a non rotating black bole. On the other hand, Penrose had shown that energy could be extracted from a rotating black bole, by a classical process. This indicated that there should be a spontaneous emission in the super radiant modes, that would be the quantum counterpart of the Penrose process. In trying to understand this emission in the super radiant modes, in terms of quantum field theory in curved spacetimes, I stumbled across the fact that even non rotating black boles, would radiate. Moreover, the radiation would be exactly what was required, to prevent a violation of the second law. Bekenstein was right after all, but in a way be hadn't anticipated.

Hence, black hole physics connects Hawking's area theorem to the second 
law of thermodynamics: $d S \geq 0$. A black hole with an event horizon of area $A$ has an entropy given by

$$
S_{B H}=\frac{k_{B} A}{4 G \hbar}=\frac{k_{B} \pi R_{0}^{2}}{G \hbar}=\frac{k_{B} A}{4 l_{P}^{2}},
$$

where $k_{B}$ is Boltzmann constant. By a sophisticated analysis of quantum fields on the curved spacetime associated to the black hole, Hawking was able to prove [6] that coherently with the thermodynamic behavior, a black hole emits a black body radiation at a temperature given by the celebrated formula

$$
T_{B H}=\frac{\hbar}{8 \pi G k_{B} M} \text {. }
$$

A layman explanation of the general philosophy underlying this basic result can be at least qualitatively appreciated if we consider the interaction of the event horizon with a quantum field. As the field approaches the horizon its fluctuations (even the virtual ones), as seen by an asymptotic Schwarzschild observer, slow down more and more as the field approaches the horizon. In particular, short-lived virtual fluctuations are stretched over the full surface horizon area (Fig. 14) and become physical fluctuations since a virtual particle that exists for an arbitrarily long time ...is an ordinary particle! Hence, black holes are not so black and through their thermal emission they should evaporate at faster and faster rate. For this to happen for standard black holes you have to wait for eons! Some numerical figures may be of help,

$$
\begin{aligned}
T_{B H}(\text { few Sol M }) & \simeq 10^{-8} \mathrm{~K} \\
T_{B H}(\text { Moon }) & \simeq 10 \mathrm{~K} \\
T_{B H}(\text { a boulder }) & \simeq 10^{18} \mathrm{~K} \\
T_{B H}\left(\mathrm{M}_{\text {Planck }}\right) & \simeq 10^{32} \mathrm{~K}
\end{aligned}
$$

Hence, Hawking temperature is tiny for ordinary astrophysical masses, but it tends to grow exponentially as we consider black holes of smaller and smaller masses. Hawking speculated that tiny cosmological black holes should exists as remnants of the Big Bang and that they could be revealed through the energetic explosion resulting from their runaway evaporation. Alas, none has been revealed up to now! It is also worthwhile to stress that black hole entropy $S_{B H}$ is proportional to the area $A$ of the event horizon and not to the 3 -dimensional volume $V$ enclosed within 


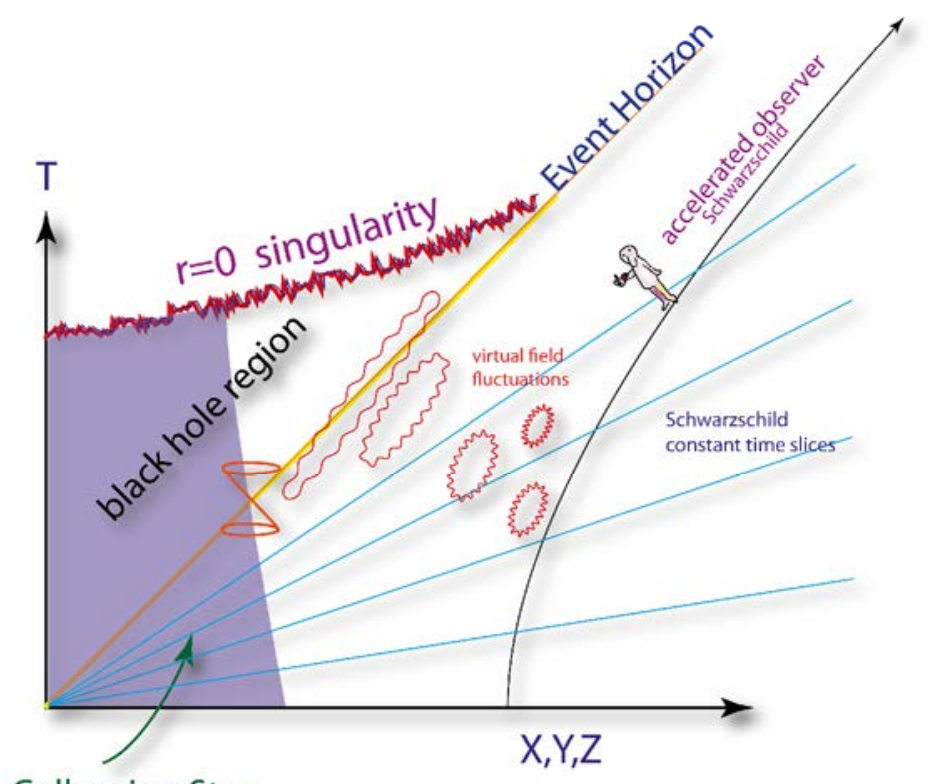

Collapsing Star

Fig. 14 - The behavior of quantum field fluctuations as they approach the borizon.

the (horizon) surface area $A$ (Fig. 15). This is a basic feature of the thermodynamics of a system involving gravity. For a usual thermodynamical system associated to a local QFT, we have $S \propto V$. In particular, the number of QFT states with a maximum energy density $\epsilon_{\max }$ that can be gathered in a $3 d$ volume $V$ typically is $N_{Q F T} \propto \exp \left[s\left(\epsilon_{\max }\right) V\right]$, where $s\left(\epsilon_{\max }\right)$ is the entropy density as a function of the energy(density). Hence, $S_{B H}$ is the maximum entropy of a region $V$ of $3 \mathrm{D}$ space: an entropy that is proportional to the surface area measured in Planck units. The basic fact remains that $S_{B H}$ is much larger than the physical (massenergy) entropy of the collapsing star generating the black hole, and one is left with the very difficult question of understanding its physical origin. Are we touching here, with the interpretative physical problems raised by Hawking radiation, the boundary between general relativity and quantum theory? One may think that the Mathematical entropy of a $\mathrm{BH}$ is a measure of the potential capacity of the $\mathrm{BH}$ to store information, or that we need the powerful extension of quantum field theory provided 


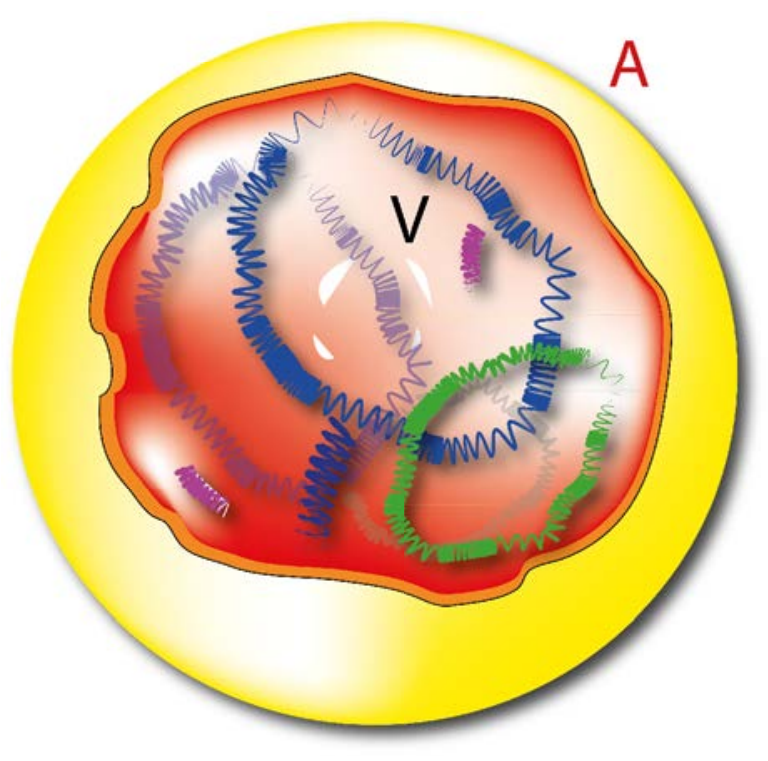

Fig. 15 - The black bole entropy $S_{B H}$ is proportional to the surface area of the event horizon.

by string theory to address a state counting interpretation of $S_{B H}$ (there have been indications in this direction) ... Still it is fair to say that we do not have a complete theory (and perhaps the right new ideas!) and Hawking radiation is challenging us, by offering a great opportunity to probe Gravity and the Quantum.

\section{Mind OVER MATter}

Stephen Hawking was arguably one of the greatest relativist of the last fifty years. In this vastly incomplete note, a written report of my short address at the Istituto Lombardo to honor his memory, we just touched upon his many impressive, if not revolutionary, contributions to our understanding of General Relativity and of their impact on the physics of the Universe. He had a public, romanticised, image, in the words of his lifelong friend Roger Penrose, the symbol of the triumph of mind over matter. An image fully justified by the fact that, notwithstanding his physical condition, he was able to establish extraordinary representations of the 
patterns of the world, which thrive on surprise and beauty.

\section{REFERENCES}

[1] S. W. Hawking, G. F. R. Ellis, Singularities in homogeneous world models Phys Lett 17, 246 (1965).

[2] S.W. Hawking, R. Penrose, The Singularities of gravitational collapse and cosmology, Proc.Roy.Soc.Lond.A A314 (1970) 529-548

[3] S. W. Hawking, Black holes in general relativity, Comm. in Math. Physics. 25 (2): 152-166. (1972).

[4] J. M. Bardeen, B. Carter, S. W. Hawking, The four laws of black bole mechanics, Comm. Math. Physics volume 31, 161-170 (1973).

[5] S. W. Hawking, G. F. R. Ellis, The Large Scale Structure of Space-Time, Cambridge University Press, (1973).

[6] S. W. Hawking, Black hole explosions?, Nature, 248 (5443): 30-31, (1974).

[7] R.P. Kerr, Gravitational Field of a Spinning Mass as an Example of Algebraically Special Metrics, Physical Review Letters 11, 237 (1963).

[8] Peter Simon, marquis de Laplace, as announced in Exposition of the system of the World, Part II, p. 305. Published proof in Allgemeine geographische Ephemeriden herausgegeben von F. von Zach. IV. Band, I. Stück, I. Abhandlung, Weimar (1799); translation in English: Hawking, Stephen W.; Ellis, George F.R. (1973). The Large Scale Structure of Space-Time. Cambridge University Press. pp. $365 \mathrm{ff}$.

[9] J. Michell, On the Means of Discovering the Distance, Magnitude, \&c. of the Fixed Stars, in Consequence of the Diminution of the Velocity of Their Light, in Case Such a Diminution Should be Found to Take Place in any of Them, and Such Other Data Should be Procured from Observations, as Would be Farther Necessary for That Purpose. By the Rev. John Michell, B. D. F. R. S. In a Letter to Henry Cavendish, Esq. F. R. S. and A.S., Philosophical Transactions of the Royal Society of London, Vol. 74 (1784), pp. 35-57 (Stable URL: http://www.jstor.org/stable/106576)

[10] J. R. Oppenheimer and H. Snyder, On Continued Gravitational Contraction, Phys. Rev. 56, 455 (1939),

[11] R. Penrose, Gravitational collapse and space-time singularities Phys. Rev. Lett. 14, 57 (1965).

[12] R. Penrose, Gravitational Collapse: The Role of General Relativity Nuovo Cimento Rivista Serie 1 (1969).

[13] K. Schwarzschild, Über das Gravitationsfeld eines Massenpunktes nach der Einsteinschen Theorie, Sitzungsberichte der Königlich Preussischen Akademie der Wissenschaften. 7: 189-196 (1916) 